\title{
Towards adaptability: taking advantage of existing tools in a LMS
}

\author{
Pedro Leiva-Chinchilla*, Jose Vega-Alvarado ${ }^{\dagger}$, Andrea Calvo-Elizondo ${ }^{\ddagger}$, Agustín Francesa-Alfaro ${ }^{\S}$ \\ TEC Digital, Instituto Tecnologico de Costa Rica \\ Cartago, Costa Rica \\ Email: $\left\{{ }^{*}\right.$ peleiva ${ }^{\dagger}{ }^{j}$ vega,${ }^{\ddagger}$ andrea.calvo, ${ }^{\S}$ afrancesa $\} @$ tec.ac.cr
}

\begin{abstract}
Each day, users look for digital tools, smarter, usable and adaptable, that make their life easier. Learning management systems with these features, are the ones that become more valuable for those who use them, but most of LMS are not flexible about the student's specific needs. This article presents an adaptative prototype, aiming to offer the users of the LMS platform better experiences related to navigation, through adaptative user interface, using existing information about each user. To achieve that, we propose the use of data obtained from applications such as the user model, the evaluation module, the calendar and the navigation history, to adapt the user interface according to the specific needs of each user. This article proposes a prototype that can be used to achieve adaptability. It was validated by 20 users using a usability test and the results show how this prototype will be useful to them.
\end{abstract}

Keywords-e-learning, adaptative, user interface, LMS, $\operatorname{dotLRN}$

\section{Introducción}

En años recientes, la tecnología adaptativa ha llamado la atención de diversos grupos de investigadores, incluyendo el área de educación. Sin embargo, para llegar a esto, es necesario conocer las características, expectativas, conocimientos y preferencias de los usuarios en las plataformas educativas [1]. Esto significa que es importante contar con un sistema que recolecte toda esta información para poder interpretarla y generar perfiles de comportamiento [2].

Actualmente con el auge de las aplicaciones móviles y simplicidad de sus funciones, los usuarios requieren cada vez más que el uso de algún servicio o herramienta sea lo más usable posible y que las interfaces se adapten a una necesidad puntual en un momento dado.

Por lo tanto, en este artículo se presenta un prototipo de interfaz adaptativa para estudiantes, con el objetivo de permitir a los usuarios de la plataforma a obtener mejores experiencias relacionadas a la navegación, por medio de la adaptabilidad, utilizando información existente de cada usuario en un Learning Management System (LMS).

Para lograr este objetivo y generar una mayor fluidez en el uso del LMS, se propone incorporar adaptaciones a partir de información del modelo de usuario, rastros de uso y el calendario de las evaluaciones.
Este último es un elemento muy importante relacionado con el proceso de enseñanza-aprendizaje, debido a que muchas veces los estudiantes cuentan con poco tiempo para entregar una tarea o realizar una actividad, así de las múltiples opciones que ofrece el sistema, el cual puede ser optimizado con una adaptación en la navegación.

Este artículo se compone de las siguientes secciones: antecedentes, trabajo relacionado, propuesta, limitaciones, conclusiones y trabajo futuro.

A continuación, se describen los antecedentes relacionados.

\section{Antecedentes}

En las plataformas LMS, existen grupos heterogéneos de usuarios, y cada uno de ellos posee características y necesidades individuales. Es por esto que la implementación de entornos adaptativos basados en sus características, su entorno, expectativas, conocimientos y preferencias, deben extenderse a las plataformas educativas [3].

Para lograr adaptar el sistema a estas preferencias, es necesario realizar un modelado, el cual es definido por [4] como el proceso de creación o actualización del modelo de usuario a partir dftae la recolección de distintos datos implícitos (obtenidos del comportamiento del usuario) y datos explícitos (solicitados manualmente al usuario).

También [5] establece dos dimensiones a tomar en cuenta en el desarrollo de un LMS adaptativo: ¿qué podría ser adaptado? y el modelo del estudiante, el cual es la base para desarrollar la adaptación.

En [6] se diferencian los conceptos de adaptabilidad, personalización y extensibilidad. El ámbito de esta investigación, estará centrado en la adaptabilidad que, de acuerdo con [7], se refiere a un sistema inteligente que automáticamente se adapta a las necesidades del usuario incluyendo: la selección de información y de servicios (contenido requerido por el usuario), la funcionalidad (funcionalidades requeridas para desarrollar tareas), la presentación de información (modalidad y codificación requerida para recibir el contenido), y la interacción humano-computador (métodos requeridos para ingresar comandos o datos y para recibir información y servicios).

En el ámbito de educación, se considera que un sistema es adaptativo si cumple con [8]: el monitoreo de las actividades de sus usuarios; las interpreta en modelos 
de dominios específicos; infiere los requerimientos y preferencias a través de actividades interpretadas, representa apropiadamente estos modelos; y finalmente, actúa sobre el conocimiento disponible de sus usuarios y sobre el tema en cuestión, para facilitar dinámicamente el proceso de enseñanza-aprendizaje. Así, sistemas adaptativos en educación pueden ser descritos como un sistema personalizado que, además del descubrimiento y montaje de contenido, es capaz de proporcionar una entrega de cursos adaptativos, una interacción y colaboración adaptativas [1].

\section{Trabajos Relacionados}

A través de los años se ha trabajado en distintas formas de adaptar el entorno de aprendizaje. Para ello se proponen herramientas conocidas como Adaptive Educational Hypermedia Systems (AEHS) y son definidos como: Hipertexto e hipermedia que reflejan algunas características del usuario en el modelo de usuario y lo aplican para adaptar varios aspectos visibles del sistema a él. En otras palabras, el sistema debe satisfacer tres criterios: debe ser un hipertexto o sistema hipermedia, debe tener un modelo de usuario y debe ser capaz de adaptarse utilizando este modelo [5].

Por su parte, existen distintos métodos para realizar esta adaptación, estos determinan cuales características del sistema son diferentes para cada estudiante. Las características de adaptación pueden ser divididas en 2 grupos: presentación adaptativa y soporte de navegación adaptativa [9]. La primera de ellas se basa en los enlaces y se caracteriza por incluir orientación directa, la adaptación de mapas, ocultar enlaces, anotación y generación de enlaces. $\mathrm{Y}$ el segundo grupo se basa en el contenido, por ejemplo, la presentación adaptable del texto [5]. Para lograr obtener este tipo de adaptación en el sistema, es necesario poseer insumos sobre la información del usuario, es por esto que nace la incógnita ¿Qué tipo de información se debe recolectar?, [5] menciona que un sistema puede proporcionar adaptabilidad mediante, el conocimiento previo de un estudiante, metas de aprendizaje, habilidades cognitivas y estilos de aprendizaje de los estudiantes, similar a como lo propone [2].

Actualmente existen AEHS que generan ajustes dependiendo de las preferencias de cada uno de los estudiantes. En la tabla 1 se pueden visualizar las herramientas AEHS que han sido desarrolladas, la información que utilizan para generar adaptabilidad y de que forma la aplican dentro del sistema.

\section{Contexto del TEC Digital}

La propuesta que se presenta, busca mejorar la experiencia que tienen los usuarios en el uso del LMS, es decir, extender el sistema, para que sea capaz de adaptarse al comportamiento y preferencias de los usuarios, similar a la manera en que lo realizan los sistemas AEHS. Actualmente el TEC, cuenta con un sistema de gestión del aprendizaje basado en dotLRN ${ }^{1}$ OpenACS [10], [11], llamado TEC

1. .LRN: plataforma LMS basada en software libre, www.dotlrn.org
Digital, el cual provee a los estudiantes y profesores de la Universidad, características como materiales de aprendizaje, quizes, foros, chats, asignaciones, entre otras.

Teniendo claro lo anterior, para llegar a cumplir el objetivo, es necesario contar con datos que describan el comportamiento y preferencias del usuario; es de este modo que se propone tomar datos de las distintas herramientas desarrolladas en la plataforma TEC Digital como insumo para generar la adaptabilidad. A continuación, se hace una pequeña descripción de las herramientas que proporcionan la información requerida para realizar adaptaciones, las cuales se analizan y seleccionan algunas en específico para el prototipo propuesto:

- Modelo de usuario (UM): herramienta que permite recolectar datos de los estudiantes en un curso utilizando mecanismos explícitos, específicamente de sus estilos de aprendizaje, necesidades de aprendizaje, información socio-académica, índice de logro, forma de comunicación, entre otros. El UM almacena las respuestas de los estudiantes para cada uno de los instrumentos, para después presentar los resultados obtenidos y de esta manera generar insumos en la toma de decisiones en el proceso de enseñanza-aprendizaje [2].

- Evaluaciones: sistema que permite a profesores realizar asignaciones como tareas, proyectos escritos y hasta exámenes, definiendo instrucciones y fechas de entrega, de modo que los estudiantes puedan enviar sus soluciones y así el profesor realiza la evaluación respectiva.

- Gestor de actividades de aprendizaje: Aplicación que permite a los profesores crear y aplicar, a los estudiantes en un curso, actividades como: cuestionarios, sopa de letras, crucigramas, ordenamientos entre otros. El UM utiliza esta herramienta para recabar los datos de los estudiantes [12].

- Marco Lógico Colaborativo: Es una aplicación desarrollada inicialmente por $\mathrm{aDeNu}$, de la UNED de España y posteriormente optimizada en el TEC Digital. Esta herramienta contribuye a que los trabajos en grupo generen un valor agregado y compromiso de parte de los estudiantes [13]; además puede proveer indicadores de colaboratividad al UM.

- TAM: paquete que tiene la funcionalidad de registrar todas las acciones que un usuario realiza sobre la plataforma del TEC Digital. Este paquete es vital para conocer los historiales de navegación de cada usuario [11].

- Calendario: servicio de calendario que se encuentra dentro del LMS y que contiene todas las fechas de los eventos y actividades de un usuario, grupo de clase o comunidad, las cuales son un insumo muy importante para añadir la temporalidad a las adaptaciones.

- AROHA: agente recomendador de objetos de aprendizaje desarrollado en el TEC Digital [14], el cual, como su nombre lo indica, recomienda objetos de 
Table 1. HeRremientas AEHS [5].

\begin{tabular}{|c|c|c|c|}
\hline Sistema & Año & Datos & Adaptatividad \\
\hline CS383 & 1999 & $\begin{array}{l}\text { Dimensión sensitiva/intuitivo, vi- } \\
\text { sual/verbal y secuencial/global de } \\
\text { FSLSM }\end{array}$ & Ordenar objetos multimedia \\
\hline MANIC & 2000 & $\begin{array}{l}\text { Combinación de preferencias de } \\
\text { aprendizaje }\end{array}$ & $\begin{array}{l}\text { Uso de texto extensible (ocultar y pre- } \\
\text { sentar contenido adicional) }\end{array}$ \\
\hline IDEAL & 2002 & Determinado por el profesor & $\begin{array}{l}\text { Ordenar, incluir y seleccionar material } \\
\text { didáctico }\end{array}$ \\
\hline MASPLANG & 2002 & FSLSM & $\begin{array}{l}\text { Adaptación en términos de elección de } \\
\text { los formatos de los medios pertinentes, } \\
\text { estrategias de instrucción y herramien- } \\
\text { tas de navegación }\end{array}$ \\
\hline LSAS & 2003 & $\begin{array}{l}\text { Dimensión secuencial/global de } \\
\text { FSLSM }\end{array}$ & $\begin{array}{l}\text { Ocultar/presentar enlaces adicionales y } \\
\text { elementos del curso }\end{array}$ \\
\hline iWeaver & 2003 & $\begin{array}{l}\text { Presentación de preferencias psi- } \\
\text { cológicas y de presentación }\end{array}$ & $\begin{array}{l}\text { Ordenar enlaces, y ocultar enlaces para } \\
\text { seleccionar modos de presentación y } \\
\text { herramientas de aprendizaje }\end{array}$ \\
\hline INSPIRE & 2003 & $\begin{array}{l}\text { Modelo de estilo de aprendizaje } \\
\text { Honey and Mumford }\end{array}$ & $\begin{array}{l}\text { Método y orden de la presentación del } \\
\text { contenido }\end{array}$ \\
\hline TANGOW & 2004 & $\begin{array}{l}\text { Dimensión sensitiva/intuitivo y se- } \\
\text { cuencial/global de FSLSM }\end{array}$ & $\begin{array}{l}\text { Orden las tareas y orden de los elemen- } \\
\text { tos dentro de la tarea }\end{array}$ \\
\hline AHA! & $2005 / 2006$ & Determinado por el profesor & $\begin{array}{l}\text { Adaptación en términos de selección de } \\
\text { artículos para presentar, ordenar y crear } \\
\text { diferentes rutas de navegación }\end{array}$ \\
\hline
\end{tabular}

aprendizaje con base en un diseño instruccional [15].

\section{Metodología}

La metodología que se siguió esta basada en una serie de actividades que se han ido desarrollando con el fin de mejorar las experiencias de los usuarios en la plataforma.

La primera, se basó en una encuesta de percepción de estudiantes y profesores con respecto a la estructura del portal del curso, donde se resaltó principalmente la necesidad de flexibilizar algunos accesos y formas de navegar y dio origen a varios proyectos, incluyendo este. Esta encuesta constaba de 7 preguntas y fue aplicada a 80 usuarios.

Con base en dichas necesidades, se realizó una revisión de todas las herramientas que podrían brindar algún tipo de información como insumo para realizar adaptaciones en la interfaz del LMS, las cuales se describen en la sección anterior: Contexto del TEC Digital.

Después de este análisis, se realizó una selección de herramientas para definir un conjunto de criterios para realizar la adaptación. A su vez se estudió a nivel de datos, la viabilidad técnica del desarrollo de una herramienta de este tipo. Una vez hecho esto, se realizó el diseño de la interfaz gráfica, tomando estos criterios de adaptación, así como de usabilidad y accesibilidad.

Por último, se valida la propuesta de este artículo, mediante un instrumento de medición basado en el Sistema de Escala de Usabilidad (SUS por sus siglas en inglés). El instrumento representa una medición de usabilidad basada en una escala de Likert, donde el usuario responde diez preguntas de un cuestionario indicando que tan acuerdo o en desacuerdo está en un nivel de 5 puntos y se aplica una fórmula para obtener el resultado [16]. Es importante mencionar que este instrumento ha sido utilizado por más de 25 años a nivel mundial en estudios de usabilidad [17].

Antes de aplicar el instrumento, el mismo fue validado en una primer fase por 3 estudiantes, estos revisaron el instrumento e hicieron observaciones a las preguntas, seguidamente se aplicó y se volvió a validar con otras 3 personas.

Finalmente, se aplicó el instrumento a 20 usuarios, ya que la intención inicial fue validar el diseño con una muestra pequeña, mediante la cual se obtuvieron los principales resultados que avalan la propuesta.

El objetivo a futuro es una vez que se ha desarrollado el módulo e instalado en el ambiente de producción, volver a aplicar el instrumento con una cantidad mayor de usuarios y así evaluar la efectividad de las mejoras aplicadas al prototipo inicial.

\section{Propuesta de adaptación del LMS}

A partir de las herramientas descritas anteriormente en la sección del Contexto del TEC Digital, se realizó un análisis de los datos almacenados por estas herramientas en cada una de sus Base de Datos, con el fin de definir una arquitectura que pueda alimentar los criterios necesarios para realizar adaptación en el LMS dependiendo de las necesidades de cada usuario de la plataforma (Figura 1).

$\mathrm{La}$ arquitectura definida provee un conjunto de datos que sirven como insumo para la adaptación de ciertos componentes que se describen a continuación:

- Basado en modelo de usuario: A través de la información recolectada por el modelo de usuario, 


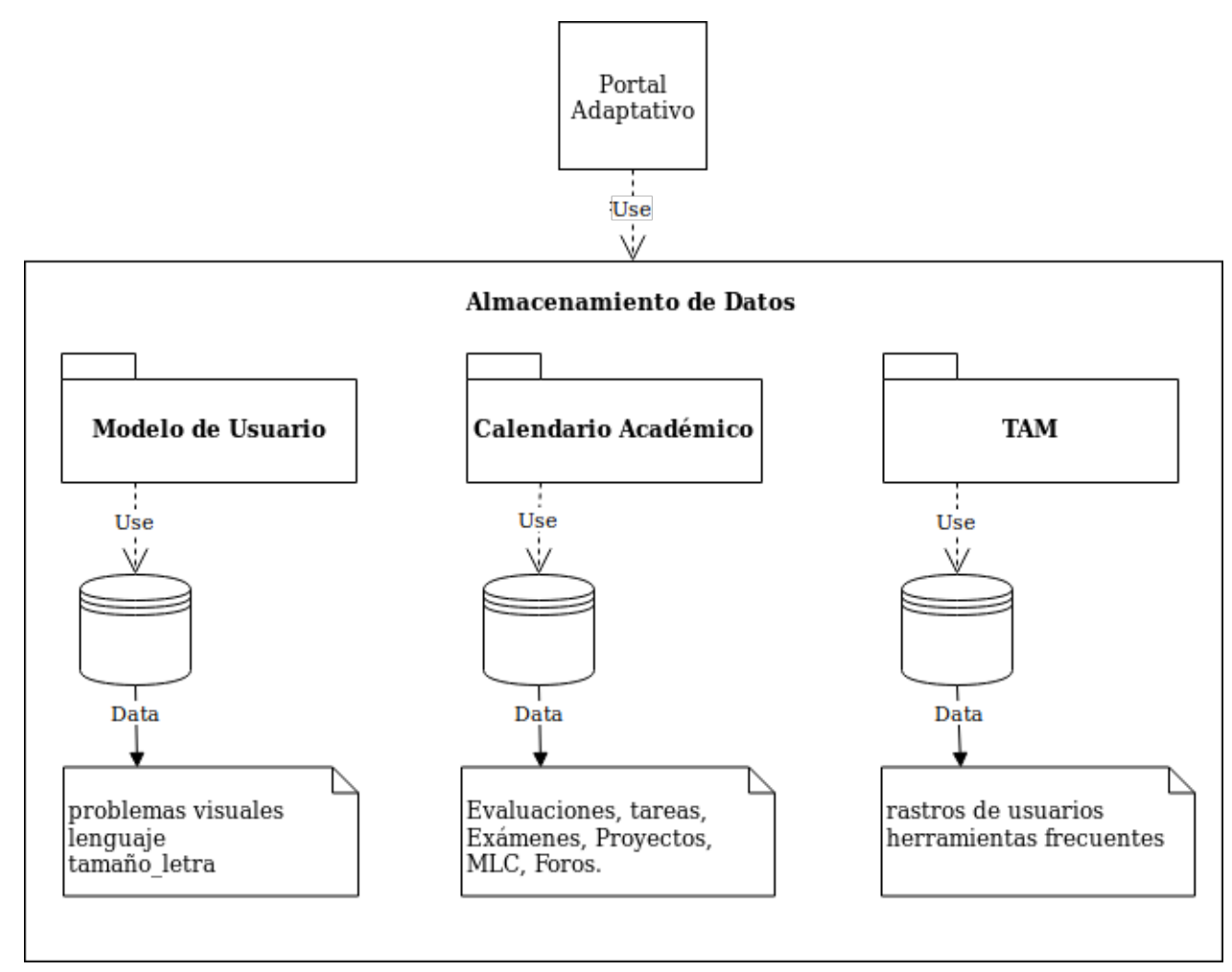

Figura 1. Arquitectura

[2]; es posible realizar la adaptación en cuanto a accesibilidad, por ejemplo, el aumento del tamaño de la tipografía, color de la fuente, eliminación o ajuste de animaciones y alto contraste figura fondo por omisión, la tipografía (sin serifas o adornos).

Además, se puede cambiar el idioma del usuario según su procedencia o información de los idiomas que maneja.

- Basado en rastros de navegación: Esta información consiste en el registro de las interacciones de los usuarios en la plataforma, captando los clics y así poder obtener conocimiento sobre sus preferencias de navegación [11]. Es decir, a través de esta información, mediante la herramienta TAM, se puede llegar a conocer las rutas más visitadas por un usuario.

Con este tipo de información, se puede generar adaptación por medio de la reorganización de los menús, así como accesos rápidos o directos a las acciones recientes, de mayor acceso o interés.

Por otro lado, se podrían generar perfiles de cada profesor, registrando las herramientas que utiliza en un curso.

- Basado en el calendario académico: La mayoría de LMS proveen un servicio o herramienta para gestionar las evaluaciones de los cursos, las cuales la mayoría del tiempo tienen una fecha límite para ser entregadas, por lo tanto, son datos que proveen información para realizar una adaptación puntual y específica en un momento determinado.

Tomando como referencia la información generada por los módulos de Evaluaciones y Calendario disponibles en la plataforma TEC Digital, es posible mostrar a los usuarios accesos directos o ventanas emergentes, que le permitan hacer el proceso de entrega de una manera directa, sin necesidad de tener que llegar a niveles más profundos de navegación o realizar el proceso completo.

Tomando en cuenta la arquitectura propuesta y los insumos disponibles, se planteó el diseño de interfaz de la página principal del TEC Digital la cual puede visualizarse en la figura 2 y que consta de los siguientes elementos:

- Actividades relacionadas al día actual: A través de los datos arrojados por la herramienta del calendario académico del usuario, se presentan todas las actividades que están programadas para el día actual. Esto permite a los estudiantes acceder de manera rápida y sencilla a sus evaluaciones y actividades de todos sus cursos en un mismo sitio, evitando retrasos que puedan presentarse por tener que navegar más a profundidad en la plataforma y a cada uno de los cursos por separado.

- Próximas Actividades: De igual manera, se presenta al usuario las actividades (evaluaciones, foros, Marcos Lógicos Colaborativos), que están próximas a entregar, de manera que el estudiante tenga visible 


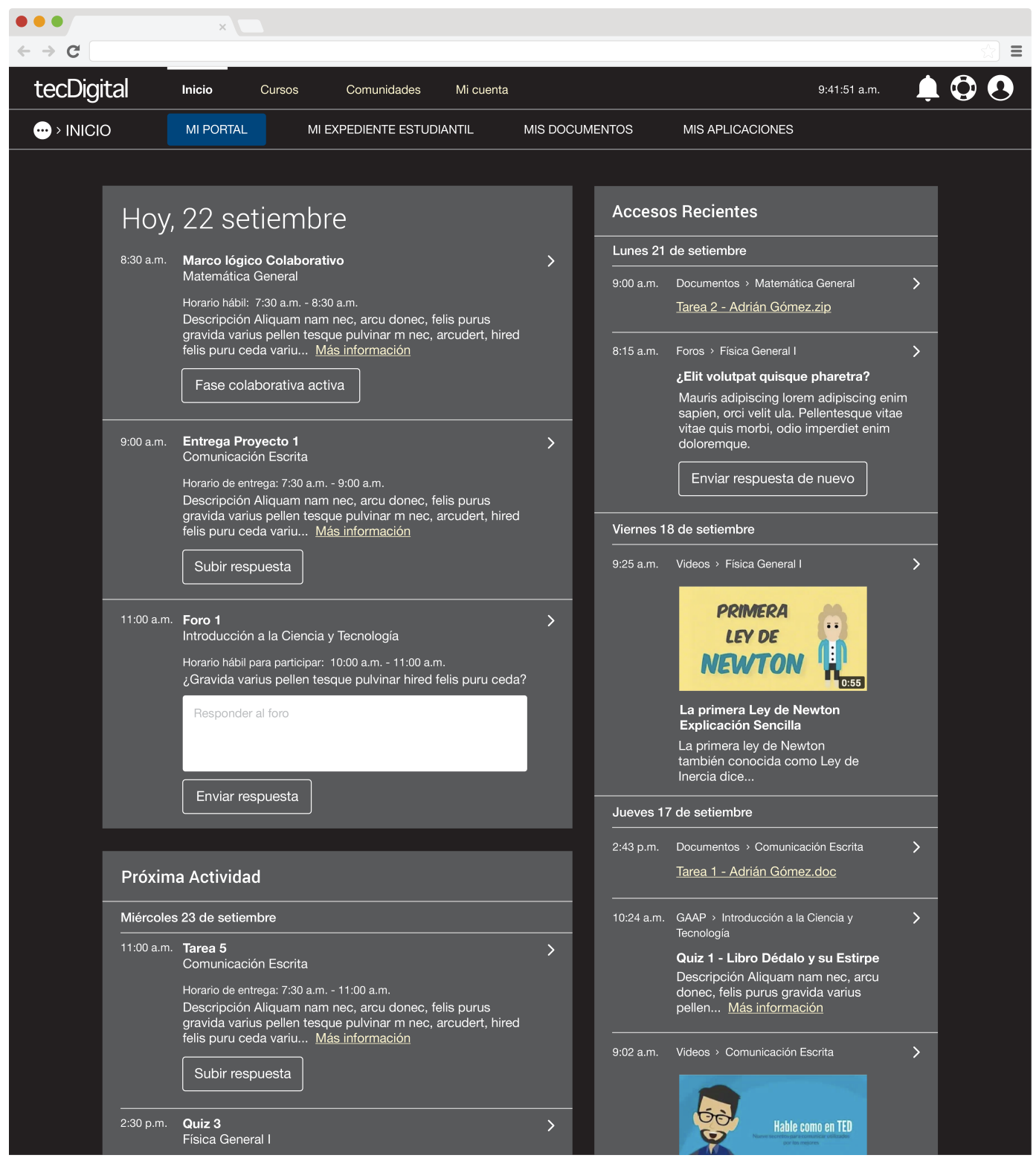

Figura 2. Propuesta de diseño en alto contraste

sus entregas, con el fin de programar adecuadamente su tiempo.

- Accesos Recientes: Por medio de los rastros de navegación que son almacenados en la plataforma, en esta sección de la interfaz se muestran todos aquellos accesos y recursos que el usuario accedió recientemente, permitiendo reducir la cantidad de clics para ingresar nuevamente a contenido de interés.

- Configuración de visualización: También se realizan ajustes automáticos relacionados a los colores en que se muestra la interfaz, tomando datos relacionados a accesibilidad por medio de la herramienta del Modelo de Usuario, la interfaz puede presentarse en colores de alto contraste o bien con los colores por defecto.

- Configuración de lenguaje: Del mismo modo a partir del Modelo de Usuario, la interfaz puede ajustarse para mostrarse en lenguaje español o inglés de manera automática.

\section{Resultados}

El instrumento utilizado fue aplicado a una muestra aleatoria de 20 estudiantes de pregrado (bachillerato y licenciatura) usuarios activos de la plataforma, 10 hombres y 10 mujeres, entre los 18 y 30 años. La dinámica empleada para que los usuarios contestaran el instrumento, se basó en mostrarles el prototipo desarrollado y una vez que hacían 
algunas inferencias personales se les presentaba el instrumento para que desarrollaran su respuesta.

Cada pregunta en este instrumento tiene una escala de calificación de un 1 al 5, siendo 1 "muy en desacuerdo" y 5 "muy de acuerdo".

La primera pregunta del instrumento hacía referencia a que tanto el usuario usaría este prototipo del sistema de manera frecuente. Los resultados obtenidos, una vez llevada a cabo la aplicación del instrumento, fue que el $80 \%$ de los encuestados consideraron que utilizarían la herramienta frecuentemente.

El $95 \%$ de los usuarios consideran útil una herramienta que presente contenido adaptable a sus necesidades tal y como la que se muestra en el prototipo. Asimismo, en promedio, los usuarios califican con un valor de 4.2 la facilidad de uso del prototipo y el $85 \%$ de los encuestados considera que las funcionalidades que se visualizan en el prototipo se encuentran bien integradas entre las mismas y con la plataforma LMS.

En relación con la percepción que tuvieron los usuarios respecto al prototipo presentado, se obtuvo que el $85 \%$ de los encuestados opinaron que estaban de acuerdo con seguir las recomendaciones que le fueron presentadas por el prototipo.

En cuanto a la pregunta " ¿Cree que necesitaría soporte técnico y/o capacitación para utilizar esta propuesta?", la calificación en promedio asignada por los encuestados fue de 2.9, lo cual indica que los encuestados consideran la interfaz lo suficientemente intuitiva, es decir, que no necesitan de capacitación o soporte técnico para utilizar el sitio. En esta misma línea, el promedio de respuesta para la pregunta planteada como "¿Considera que necesita aprender mucho sobre las funcionalidades que se visualizan en la propuesta antes de que pueda utilizarlo efectivamente?"fue de 2.6, es decir, no es difícil de aprender.

Finalmente, el puntaje obtenido en la escala SUS, una vez procesados los datos del instrumento aplicado, es cercano a aceptable [18].

\section{Conclusiones y Trabajo Futuro}

Se ha propuesto un enfoque para extender un LMS con funcionalidades adaptativas que buscan disminuir el tiempo de interacción de los estudiantes y profesores; todo ello con el objetivo de mejorar la eficiencia en el desarrollo tareas clave y así la experiencia de los usuarios del LMS.

Un elemento a considerar, es que muchos LMS son de software libre, por lo que esta propuesta da la posibilidad a que otras instituciones puedan implementar una solución parecida a la descrita en este artículo, ya que mucha de la información que se utiliza ya existe en el LMS.

Por otro lado, es muy importante tomar aspectos de usabilidad en los LMS, ya que es una herramienta que utilizan diversos tipos de usuarios, de diversas áreas, edades y nivel tecnológico, por lo que, una interfaz adaptativa que facilite el uso, es un elemento de valor para los usuarios.

Sobre esa línea, los elementos de accesibilidad deben ser tomados en cuenta, sobre todo en adaptaciones, de manera que los usuarios con algunas dificultades o discapacidades puedan con solo una configuración previa, que el sistema se adapte a dichas necesidades y así su experiencia en el uso de la plataforma sea la mejor.

Sobre la metodología, el uso de un instrumento validado para recabar información es un elemento de gran valor en una propuesta como esta, ya que permite conocer la aceptación y utilidad de un producto antes de realizar las etapas de desarrollo e implementación.

Finalmente, como se pudo evidenciar en la aplicación del SUS, un factor a tomar en cuenta en este desarrollo, es la elaboración de las ayudas para los usuarios, esto debido a que no están acostumbrados a este tipo de adaptaciones en sistemas LMS.

En cuanto a trabajo futuro, la adaptación que se espera empezar a realizar de manera más inmediata, es la relacionada al calendario y evaluaciones, pues es la que más beneficios daría, sobre todo a los estudiantes.

Además, se espera que una vez desarrollada esta funcionalidad se realice nuevamente el SUS con el fin de generar más datos que permitan una retroalimentación de los usuarios.

Por último, con base en las experiencias recolectadas, se esperan generar más propuestas de adaptación basadas en información existente en la plataforma, que, con un tratamiento adecuado, se materialicen en valor para los usuarios.

\section{Referencias}

[1] M. Despotovic-Zrakic, A. Markovic, Z. Bogdanovic, D. Barac, and S. Krco, "Providing Adaptivity in Moodle LMS Courses," Journal of Educational Technology \& Society, vol. 15, no. 1, pp. 326-338, 2017. [Online]. Available: http://www.jstor.org/stable/jeductechsoci. 15.1 .326

[2] M. Chacón-rivas, O. C. Santos, J. G. Boticario, T. E. C. Digital, I. Tecnológico, D. C. Rica, and C. Rica, "Modeling Learner Information within an Integrated Model on Standard-Based Representations," Proceedings of the 5th Workshop on Personalization Approaches for Learning Environments (PALE 2015)., pp. 31-38, 2015.

[3] P. Brusilovsky, "Adaptive hypermedia," User Modeling and UserAdapted Interaction, vol. 11, no. 1-2, pp. 87-110, 2001.

[4] C. Carmona, G. Castillo, and E. Millán, "Discovering student preferences in e-learning," CEUR Workshop Proceedings, vol. 305, pp. 33-42, 2007.

[5] S. Graf, "Adaptivity in Learning Management Systems Focussing on Learning Styles," Proceedings of the 2009 IEEE/WIC/ACM International .., vol. 3, no. December 2007, pp. 235-238, 2009. [Online]. Available: http://ieeexplore.ieee.org/lpdocs/epic03/wrapper.htm?arnumber= 5284970\{\\%\}5Cnhttp://dl.acm.org/citation.cfm?id=1632300

[6] S. Graf and B. List, "An evaluation of open source e-learning platforms stressing adaptation issues," Proceedings - 5th IEEE International Conference on Advanced Learning Technologies, ICALT 2005, vol. 2005, pp. 163-165, 2005.

[7] R. Oppermann, "From User-adaptive to Context-adaptive Information Systems (Von benutzeradaptiven zu kontextadaptiven Informationssystemen)," I-COM Journal of Interactive Media, 2009.

[8] A. Paramythis and S. Loidl-Reisinger, "Adaptive learning environments and e-learning standards," Electronic Journal of Elearning, vol. 2, no. 1, pp. 181-194, 2004. 
[9] P. Brusilovsky and E. Millán, "User Models for Adaptive Hypermedia and Adaptive Educational Systems," The Adaptive Web, pp. 3-53, 2007. [Online]. Available: http://link.springer.com/10.1007/ 978-3-540-72079-9\{\\%\}7B $\{\backslash\}\}\{\backslash \%\} 7 D 1$

[10] R. a. Calvo, E. Ghiglione, and R. Ellis, "The OpenACS e-learning infrastructure," Proceedings of the 9th Australian World Wide Web Conference, no. April, pp. 175-183, 2003. [Online]. Available: http://www.usyd.edu.au/SCH/courses/showcase/ showcase2003/sc $\left\{\backslash_{-}\right\}$files/calvo.pdf

[11] J. Couchet, O. C. Santos, E. Raffenne, and J. G. Boticario, "The Tracking and Auditing Module for the OpenACS Framework," 7th OpenACS/.LRN Conference. International Conference and Workshops on Community based environments, pp. 82-87.

[12] M. Fallas-hidalgo, J. Serrato-romero, C. Garita, and M. Chacón, "LEARNING ACTIVITIES MANAGER FOR E-LEARNING PLATFORMS," in LACLO 2013 - Octava Conferencia Latinoamericana de Objetos y Tecnologías de Aprendizaje, Valdivia, Chile, 2013. [Online]. Available: http://www.laclo.org/papers/index.php/laclo/article/view/89

[13] J. V. Alvarado, A. F. Alfaro, M. C. Rivas, and C. G. Rodr??guez, "Collaborative logical framework: An e-learning assesment tool in.LRN platform," Proceedings - 2016 11th Latin American Conference on Learning Objects and Technology, LACLO 2016, 2016.

[14] J. Solís, M. Chacón-Rivas, and C. Garita, "Agente Híbrido Recomendador de Objetos de Aprendizaje," IX Conferencia Latinoamericana de Objetos y Tecnologías de Aprendizaje, pp. 290-301, 2014.

[15] A. Francesa-Alfaro, P. Leiva-Chinchilla, M. Chacón-Rivas, and C. Garita, "User-friendly instructional design tool to facilitate course planning," Proceedings - 2016 11th Latin American Conference on Learning Objects and Technology, LACLO 2016, 2016.

[16] Brooke J., "SUS-A quick and dirty usability scale," Usability evaluation in industry, pp. 4-7, 1996.

[17] J. Brooke, "SUS : A Retrospective," Journal of Usability Studies, vol. 8, no. 2, pp. 29-40, 2013. [Online]. Available: http://uxpajournal.org/sus-a-retrospective/

[18] A. Bangor, P. Kortum, and J. Miller, "Determining what individual SUS scores mean: Adding an adjective rating scale," Journal of usability studies, vol. 4, no. 3, pp. 114-123, 2009. [Online]. Available: http://66.39.39.113/upa_publications/jus/ 2009may/JUS_Bangor_May2009.pdf 